\title{
Aspectos léxicos del Libro de los grados de las espeçias e de las yervas
}

\author{
JESÚS PENSADO FIGUEIRAS \\ Universidade da Coruña
}

\section{INTRODUCCIÓN}

El Libro de los grados de las espeçias e de las yervas (en adelante Libro de los grados), traducción parcial al castellano del Liber de simplicium medicinarum virtutibus (desde ahora lib. simp. med. virt.), atribuido mayoritariamente a Johannes de Sancto Pau$1 \mathrm{o}^{1}$, ha sido localizado en los folios 82v-87r del manuscrito II-3063 de la Real Biblioteca, de Madrid $^{2}$. La dificultad de su lectura y sus numerosas deturpaciones han motivado que no se haya catalogado y que permaneciese inédito hasta el momento en que se ha podido reconocer su fuente latina. El tratado enumera una serie de simples de origen animal, vegetal y mineral, describiendo en una primera parte (cap. 2-17), el grado en que cada elemento posee alguno de los cuatro temperamentos básicos: calor, frialdad, sequedad y humedad. La segunda parte (cap. 18-34) retoma buena parte de los mismos simples y añade otros, clasificándolos en esta ocasión según ciertas propiedades terapéuticas — «las que abren», «las que alinpian las llagas», «las que llagan el cuero», etc.- - respetando en cada capítulo una idéntica estructura: descripción de cada virtud terapéutica seguida de la enumeración de simples que poseen la propiedad en cuestión. La obra se trunca de forma brusca en el capítulo 34, del que solo se conservan tres líneas ${ }^{3}$.

Al conservar un único testimonio de este tratado, el análisis textual es fundamental para emitir alguna conjetura sobre su génesis y difusión. En concreto, el estudio léxico permite sospechar con cierto fundamento que la versión conservada no es traducción directa del latín, sino una copia de otro texto castellano anterior, ya que algunos de sus errores solo se comprenden partiendo de un término castellano previo. A partir de esta evidencia, es posible afirmar que el tratado conoció un número incierto de copias y que la conservada podría situarse en un estadio secundario o incluso terciario de la transmi-

${ }^{1}$ La edición de la obra latina corrió a cargo Kroemer (1920). Reseña de esta obra en Sarton, y Gilpatrick (1922). Aunque la atribución a este autor no es definitiva, se incluye en una breve biografía de Johannes de Sancto Paulo en Glick, Livesey (2005). Los datos actualizados de lo que se conoce de su biografía pueden consultarse en Martín Ferreira y García González (2010) y en García González (2012).

${ }^{2}$ Beaujouan (1972) no menciona esta obra en su catálogo. Tampoco forma parte de la descripción de este manuscrito en el catálogo de la Real Biblioteca (http://realbiblioteca.patrimonionacional.es/cgibin/koha/opac-detail.pl?biblionumber $=84350$, consultado en diciembre de 2012).

${ }^{3}$ Estoy ultimando la edición de este tratado, que será publicada próximamente, con el título «El Libro de los grados de las espeçias e de las yervas: edición crítica». 
sión textual ${ }^{4}$. También se podrían emitir dudas a la hipótesis de que el castellano es el idioma original al que se tradujo el texto latino, aunque no se hayan localizado copias en otras lenguas peninsulares. La razón de esta reticencia se apoya en que el texto presenta ciertos catalanismos como argela y uylo (vid. infra), que permitirían suponer la existencia de una hipotética traducción primera al catalán, hoy desaparecida. No obstante, este criterio léxico no es suficiente para fundamentar hipótesis al respecto, pues los ya citados catalanismos coexisten con otros términos como alfóstico o formiento más comunes en la zona occidental de la Península Ibérica ${ }^{5}$.

Además de estas consideraciones generales, el estudio léxicográfico aporta ciertos elementos de discusión sobre algunos étimos que presento seguidamente, en concreto en lo que respecta a su uso en esta obra y a su posible significado, que en ocasiones no coincide con las acepciones recogidas en diccionarios generales o especializados. En cualquier caso, su análisis contribuye a una mejor comprensión del Libro de los grados.

\section{ESTUDIO LÉXICO}

\section{ALCARAVÁN}

De las vmidades en el primero grado [...] De las carnes: la del puerco, carnero, pollos, anades, palominos, alcaravanes, ansares, faisanes, codornizes, pezes cochos (84vb).

Los diccionarios coinciden en definir el término alcaraván como un ave de tamaño mediano, cuya especie común presenta el nombre científico de Burhinus oedicnemus ${ }^{6}$. No obstante, el vocablo se usa en el tratado que nos ocupa para traducir el latino strutionum, en un pasaje casi paralelo en latín y castellano ${ }^{7}$. La relación entre ambos términos parece provenir de alguna dificultad en la interpretación del vocablo STRUTHIO -ONIS. Su definición según Isidoro de Sevilla parece referirse, efectivamente, al avestruz (Isidoro 1994: 108): «Struthio Graeco nomine dicitur, quod animal in similitudine avis pinnas habere videtur ; tamen de terra altius non elevatur». Pero ya Plinio el Viejo se refería al avestruz denominándola sistemáticamente struthocamelus $(\mathrm{NH}, \mathrm{X}$, 1, 29, 73; NH, XI, 47, 56), acaso para evitar confusiones con la fruta llamada struthium (Cydonia vulgaris: $N H, \mathrm{XV}, 10)$. Asimismo, los bestiarios latinos reflejan una alternancia de términos para referirse al avestruz (Vermeille 2006: 104 y ss.) ${ }^{8}$. De los textos analizados por Vermeille, solo la que él denomina «versión B» (ed. Carmody) contiene el capítulo Asida, que interpreta como autruche. Las versiones más recientes, bajo el nombre de Dicta Chrysostomi, también incluyen el capítulo De assida, al igual que la

\footnotetext{
${ }^{4}$ Analizo los aspectos generales y las fuentes de esta obra en mi comunicación titulada «En torno a la edición crítica del Libro de los grados de las espeçias e de las yervas», que será publicada próximamente en las Actas del 50 aniversario de la Asociación Internacional de Hispanistas.

${ }^{5}$ Cfr. García de Diego 1978: 46 y 153; Díaz González 1986: 206; García Arias 2007: 222-223.

${ }^{6}$ Así figura, por ejemplo, en el glosario a la edición del Libro de la caça de las aves (López de Ayala 1986: 214).

7 «Carnes: Caro porcina, pecorina, pullina, anatum, columbarum, strutionum, anserum, fasianorum, coturnicum, piscium recentium». No existen divergencias en los manuscritos colacionados por Kroemer.

${ }^{8}$ Extracto de este trabajo los datos que expongo a continuación.
} 
versión breve del Bestiaire de Pierre de Beauvais ${ }^{9}$. Por su parte, la obra De bestiis et aliis rebus (libro III) modifica este capítulo y lo denomina De assida seu struthione; igualmente, el Bestiaire divin de Guillaume le Clerc de Normandie, en su versión del Manuscrit B. M., Royal 2 C. $x i i^{10}$, contiene el capítulo De assida et strucione.

La edición de uno de los bestiarios analizados por Vermeille, el Physiologus, ya había sido realizada por Gustav Heider a partir de una versión del siglo XI. En el capítulo De Assida dice lo siguiente (Heider 1850: 578):

XXIV. De Assida.

Item est animal quod assida dicitur. quodque greci strucionem nominant. De strucione isto heremias propheta dicit. et assida in celo cognovit tempus suum.

El mismo editor indica en nota otra versión para esta cita de Jeremías (Jer. 8: 7): «milvus in coelo cognovit tempus suum». Asimismo, la Nova Vulgata publicada por el Vaticano ${ }^{11}$ presenta una nueva variante de esta misma cita: «Etiam ciconia in caelo novit tempus suum». Así pues, la cita del Physiologus presenta versiones con tres aves diferentes: assida (avestruz), milvus (milano), ciconia (cigüeña). André (1967: 147) se muestra definitivo a este respecto al afirmar que struthio traduce el griego $\sigma \tau \rho o v \theta$ óc, pero el hebreo bat ya 'ănāh, según el contexto en que aparece en la Biblia, no denomina un avestruz sino un ave rapaz nocturna, aventurando la posibilidad de que hiciese referencia al buho real. Esta variedad referencial parece indicar que el mundo medieval no identificaba inequívocamente el étimo strutium / strucium con el avestruz; además, es llamativo que este pasaje de Jeremías se traduzca de forma sistemática al romance como cigüeña ${ }^{12}$.

En cuanto al étimo alcaraván, el Diccionario de Autoridades ya lo describe como presa común en la cetrería, citando a Jerónimo Cortés: Cazan los Neblies con grande ánimo muchas suertes de aves, como son perdices, alcaravánes, lechuzas, milanos ${ }^{13}$. Fradejas Rueda (2002: 144, 147) también menciona este volátil y lo identifica con el nombre científico ya citado Burhinus oedicnemus, ave que mide entre 40 y $45 \mathrm{~cm}$. de longitud (Juana Aranzana 2000: 76). No obstante, el volumen dedicado a las aves de la Encyclopedia metódica (1788: 169-170) dedica un largo artículo al alcaraván, que también denomina Butorio y Garza dorada, entre otros apelativos ${ }^{14}$ :

\footnotetext{
${ }^{9}$ La versión larga presenta el capítulo «De l'ostruche».

${ }^{10}$ El capítulo de la versión canónica se denomina «Ostrice».

11 URL: http://www.vatican.va/archive/bible/nova_vulgata/documents/novavulgata_index lt.html (consultado en noviembre de 2012).

${ }^{12}$ Esta misma confusión terminológica ya figura en la completa descripción del struthocamelus realizada por Konrad Gesner (1555: 709 y ss.).

${ }^{13}$ La cita completa es (1672: 440): Caçan los Neblis con grande animo y diligencia muchas suertes de aves, como son Perdizes, Alcaravanes, Lechuzas, Milanos y Atavardados, Cigueñas, Saureles, Dorales, Garçotas, Paviotas y Martinetes.

${ }^{14}$ También recibe los denominativos: «pella», «ardea» $\mathrm{y}$ «buey de pantano».
} 
El alcaravan ó garza dorada es del género XXXI. ó del de la garza. Tiene dos pies y cinco pulgadas de largo desde la punta del pico á la de la cola: tres pies, diez pulgadas y algunas lineas de vuelo [...] sus piernas á proporcion son mas cortas que las de la garza: su cuerpo es mas corto, mas carnoso, y mas fornido [...] En tiempo de Bellon era muy apreciable la carne de estas aves, y pasaban por un manjar excelente; mas hoy en dia no se aprecian.

Cuatro años antes, Bru de Ramón dibujaba y describía esta ave, atribuyéndole el nombre científico ardea stellaris (1784: 13) ${ }^{15}$. El DCEH parece apuntar en esta misma dirección en las definiciones de 'árdea' y 'bitor' ${ }^{16}$. La vaga definición del $D H^{17}$ confirma que el uso del vocablo alcaraván implicó imprecisiones difíciles de desentrañar en el actual estadio de la investigación. En cualquier caso, ninguna de ellas parece indicar una relación con el avestruz, por lo que se mantiene una zona de sombra sobre la interpretación de este pasaje, tanto en su versión latina como en la castellana.

\section{ARGELA}

De las frias en el primero grado: De las veneras: boliarmenico, chimolea, argela, azero, plata, catimia, argento (83va).

Este término, que traduce el latino argilla en el lib. simp. med. virt., existe con el sentido de 'arcilla' en occitano y catalán medieval ${ }^{18}$, así como en varios topónimos catalanes de idéntico origen $^{19}$. Aunque el manuscrito presenta agrela, evolución etimológicamente posible a partir del latín ARGILLA, no he encontrado ningún documento que lo relacione semánticamente con la arcilla. En gallego se utiliza agrela y agrelo como diminutivos de agra y agro. Agrela también se identifica en castellano como una planta de uso en las boticas (Pensado Tomé 1974: 87; Colmeiro I: 577). Con esta acepción, Pensado Tomé remite al término catalán agrella, con el significado de 'acedera' o 'acederilla' $(D C V B ; D I E C 2)^{20}$. Pero la localización precisa de este vocablo en las vene-

${ }^{15}$ La página web worldbirdinfo.net indica que el ardea stellaris pertenece a la familia de los $B o$ taurus, denominados avetoro en castellano, con cuatro subespecies: avetoro mirasol (Botaurus pinnatus), avetoro lentiginoso (Botaurus lentiginosus) y avetoro australiano (Botaurus poiciloptilus), avetoro común (Botaurus stellaris). Los tres primeros se localizan en los continentes americano y australiano. Siguiendo esta denominación y como hipótesis inicial para futuras investigaciones, la que Bru de Ramón denomina «garza dorada o alcaraván» podría hacer referencia con el ave que hoy se conoce como avetoro común, cuyo tamaño y características parecen coincidir con lo indicado en la Encyclpedia metódica. Fuentes:

http://www.birdlife.org/datazone/sowbsearchresults.php?a=ns\&SearchTerms=botaurus (consultado en noviembre de 2012); http://worldbirdinfo.net/Pages/BirdCitationView.aspx?BirdID=31660\&Source=\%2FPages \%2FBirdsSearch.aspx\%3FBirdField\%3D0\%26BirdSearch\%3Dbotaurus (consultado en noviembre de 2012).

${ }^{16}$ «Árdea, 'garza, alcaraván', tomado del lat. arděa íd.»; «BITOR, 'alcaraván', del fr. butor íd., emparentado con el lat. BUTIO, quizá descendiente de una forma BUTI-TAURUS del latín vulgar, en combinación con TAURUS ‘toro', que también se ha empleado para designar el mismo pájaro». En la entrada garza también se alude la glosa «carduelus: alcaravan» en los glosarios de Castro. Spitzer intenta explicarlo insinuando que hubo cruce con carduelis ‘jilguero', conjetura que el DCEH considera «inadmisible».

17 «Nombre que se da a distintas aves zancudas de los géneros Oedicnemus, Burhinus y Charadrius, etc. Su plumaje es de coloración parda con el vientre blanco, sus patas son amarillas y tiene grandes ojos».

${ }_{18}^{18}$ Cfr., por ejemplo, Pere Hispà (1892: 28).

${ }^{19}$ Amigó (1999: 121).

${ }^{20}$ Alemany (1917) también incluye este étimo en su diccionario. 
ras, es decir, en los minerales, parece excluir la referencia a una planta. Por consiguiente, es probable que el término agrela del manuscrito sea una deturpación a partir del étimo catalán argela, que introduce la conjetura de que la traducción pudo haber sido realizada en la zona oriental de la Península Ibérica e incluso que este texto hubiese sido traducido de una hipotética versión previa en catalán.

\section{BASILICON}

De las calientes en el terçero grado. [...] De las raizes: meu, ditamo, cipero, puerros, eleboro, hermodatil, zedoarii, serpent(er)aria, bretonica, gengibre, çentaurea, basilicon, piper longo, staphisagria (83ra).

El basilicon no presenta correlato en el texto latino, al igual que el «piper longo» y la «staphisagria», por lo que su significado es oscuro. Gutiérrez Rodilla (2010: 66) menciona este vocablo y lo define como «ungüento amarillo» ${ }^{21}$, acepción que no parece tener sentido en el texto que nos ocupa, al tratarse de un compuesto. El estudio del étimo Basilicum realizado por Carrera de la Red en su edición del Dictionarium medicum de Nebrija (2001: 191) presenta una descripción mucho más amplia:

Desde el griego este término designa, por una parte, una especie de planta odorífera (ARIST. Plant. 1,3,10) y por otra, una clase de ungüento (AlEX. TRALL. 3,175). Las designignaciones tardías medievales de este tipo de planta son diversas: 1) La albahaca (Ocimum basilicum L. O. monachorum y O. minimum L.) (Diosc. 2,158. Constant. Afric. theor. 5,103, p. $24 \mathrm{a}^{\mathrm{v}}$ basilicon calidum este et siccum) ${ }^{22}$. 2) La $\neg$ gentiana. 3) La $\neg$ buglossa (GLOss. III 550, 67 St.-S. buglossa vel basilicon ossenzunge). 4) La semilla de la mejorana (ALPHITA ii p.18, 23 basilicon semen est maiorane ${ }^{23}$, ozimus idem). 5) En Plinio se utiliza para denominar una clase de nuez (nat. 15,87).

Además de las hierbas referidas, este término podría hacer referencia a lo que Serapión denomina gariofilus en el Liber aggregatus (1525: f. $115 \mathrm{v})^{24}$, incluyéndolo entre los simples cálidos y secos en tercer grado; el Liber de gradibus también lo menciona (Israelí 1515: f. 81r) ${ }^{25}$, aunque entre los simples cálidos en segundo grado. Montero Cartelle (1983: 193) se detiene a comentar este término:

${ }^{21}$ El término figura en un glosario al final del Thesoro de la verdadera Cirugía y vía particular contra la común, de Hidalgo de Agüero (1604).

${ }^{22}$ La glosa semántica es clara en el Libro del arte de las comadres, mencionando la mejorana en la misma receta, junto con el romero: «Recipe foliorum \& seminum basiliconis que es albahaca/ romero/ mayorana» (Carbón (1997, f. 80r). Lo mismo sucede en Cirugía: «Resçibe musco. gengibre \& vayas de laurel. majorana. \& calamento. \& basilicon. \& çentaurea mayor...»: Herrera (s.d., f. 167v). Para Ruyzes tampoco existe duda a este respecto: «Ozimum basilicon, la aluahaca, según algunos, fina y loca, y la que güele a claueles y a torongil» (Ruyzes de Fontecha 1999: 158)

${ }^{23}$ También en Herrera y González de Fauve (1997, f. 145r): «El gordon añade en ellos la maoirana basilicon».

${ }^{24}$ Los dos primeros tratados del Liber aggregatus de Serapión presentan semejanzas llamativas con el lib. simp. med. virt., a pesar de la considerable distancia temporal que separan la copia más antigua del texto editado por Kroemer (s. XII) y Simon Januensis, traductor latino de Serapión, que vivió entre los siglos XIII y XIV.

${ }^{25}$ Algunos estudiosos consideran que este Liber de gradibus, traducido por Constantino el Africano, es la fuente principal del lib. simp. med. virt. 
Nótese que cariofilatum puede designar al ocimum en varias de sus especies [...], aunque para ello se usa normalmente basilicon y también el Geum urbanum L. o Valeriana officinalis L. comúnmente llamada benedicta. Laguna también recoge esta denominación en su comentario al «ocimo» (Lag., II, 130)

De ser así, y siguiendo a Montero Cartelle, el abanico de referencias del basilicon se extiende a la albahaca, la genciana, la buglosa y la mejorana, además del posible clavero o giroflé (gariofilus) presente en los textos de Constantino y Serapión. La referencia a «una clase de nuez», mencionada anteriormente por Carrera de la Red podría apoyar tal hipótesis, pues este es el significado del término griego karuon, que compone el término caryophyllon que evolucionaría posteriormente en el sustantivo giroflé ${ }^{26}$. Finalmente, los dos autores mencionados recogen también el ozimo gariofilato: Serapión lo incluye entre los cálidos y secos en segundo grado, si bien precisa «in fine secundi gradus» (1525: f. 144r); por su parte, el Liber de gradibus lo describe como cálido en primer grado y seco en segundo (Israelí 1515: f. 79v). En conclusión, la aparición de este término sin correspondencia latina en la edición de Kroemer no parece ser resultado de una deturpación textual por contaminación o por desplazamiento de términos de otros pasajes; al contrario, su concurso, al igual que el piper longo y la staphisagria, parece deberse a una modificación consciente de un copista con conocimientos farmacológicos en algún momento de la difusión textual en latín o en romance.

\section{CATIMIA}

De las frias en el terçero grado [...] De las veneras: yeso, espodio, [ca]timia, ferrugo (83vb).

Aunque el manuscrito presenta timia y no figura en la fuente latina, es posible que se refiera al simple cathmia ${ }^{27}$, término que remite a la cadmía: «Sublimado metálico adherido a una chimenea o a la bóveda de un horno» $(D R A E)^{28}$, que ya aparece en el Libro de los grados en los apartados «frias en primer grado» y «humedas en segundo grado». La atribución de dos grados distintos de frialdad al mismo simple, el primero y el tercero, podría ser consciente: el Pantegni, De Practice (Israelí 1515: f. 74r) recoge dos tipos de esta escoria del metal, que en Bernardo de Gordonio se concretan como «catimia de oro \& de plata» ${ }^{29}$. De esta manera, el texto castellano utilizaría el mismo vocablo para referirse a dos elementos distintos, con grados de frialdad diferentes. No obstante, la edición de Kroemer no recoge este simple en el capítulo latino de los fríos en tercer grado, por lo que también es verosímil que su presencia sea resultado de una deturpación textual, bien de alguna familia del texto latino de la que surgió la traducción romance, bien de la difusión castellana posterior.

\footnotetext{
${ }^{26}$ Cierto es que Bernardo de Gordon menciona en una receta el «basilicon» y los clavos de girofle por separado: «...mirra \& finojo \& basilicon anis bol armenico clauos de girofre cominos eupatorio...»: Gordonio $(1989$, f. 184v). Para una idea más precisa de la complejidad del término basilicon, cf. Santamaría Hernán$\operatorname{dez}($ 2009: 97-119).

${ }^{27}$ Existen numerosos testimonios de catimia en castellano, por lo que mantengo esta opción en lugar de «cathmia». Por el contrario, no he localizado ningún otro testimonio del término timia con el significado referido.

${ }^{28}$ El DETEMA reproduce la definición de Laguna. No la tomo aquí como referencia porque alude al «hollín del cobre», cuando otros textos aluden a la catimia para denominar el sublimado de otros metales.

${ }^{29}$ Gordonio (1989, ff. 24v, 33r, 33v, 144v, 183r).
} 


\section{DRAGONDION}

De las laxatibas melecinas [...] De las que purgan la flema. Son estas: zarcacola, lacterio, sauco, anacardo, alazor, coconidium, macis, laureola, coloquintida, turbit, timi, estafizagria, hermodatilis, pelitre, euforbio, tapsia, sal gema, dragondion (85rb).

Probable evolución del helenismo dracontion para referirse al dracunculus o dragontea. Este término figura entre los simples que purgan la cólera, aunque en el texto latino del lib. simp. med. virt. se precisa claramente: «Draguintea grossos flegmaticos humores purgat». Así pues, es probable que el término aparezca desplazado en el manuscrito, como sucede con numerosas voces en otras partes del tratado.

La variante más cercana fonéticamente al vocablo analizado es dragontion, que se testimonia en catalán medieval ${ }^{30}$ y en francés clásico ${ }^{31}$. En castellano, no he podido localizar el mismo vocablo; los términos más cercanos se localizan en Colmeiro (V: 196) y Font Quer (2002: 961), quienes presentan dracontio como sinónimo castellano de la «dragontea» (Arum dracunculus L.). Ya más alejado fonéticamente, el término dragontia se documenta en $F I E$ (f. $101 \mathrm{v})^{32}$ y en TRM (f. $\left.28 \mathrm{v}\right)^{33}$, al igual que en italiano clásico; draguntia $a^{34}$ presenta también testimonios en GIL (f. 8v) ${ }^{35}$ y en TES (f. $\left.25 \mathrm{v}\right)^{36}$. Asimismo, el DETEMA recoge otras denominaciones de la dragontea menos cercanas en su fonética al término que nos ocupa.

\section{ESTRINCO}

De las secas en el segundo grado. Son estas que se siguen: asensio, fumosterre, menta, eupatorio, amomo, afrodillos, alcaparras, rauanos, virga pastoris, centaurea, pentafilon, estrinco, catapucia, ciperis, mestranto, sisilion, uva bolpicia, aneldo, puerros, coriandro (84ra).

\footnotetext{
${ }^{30}$ Ejemplo de ello es la versión catalana del Libre de les medicines particulars, editada por Faraudo de Saint-Germain (Wafid 1943: 59): («dix que la dragontina es de .ij. maneras: la .ja. a nom aran. E 1 altra dragontion. Mas 1 aran a la ffulla en ssemblant del dragontion, mas a la menor .j. poc... Mas lo dragontion a major fulla e ab senyals blancs que ssembla despulla de serp, e es de moltes colors e fa la cana grossa e ab los caps en ssemblant de rraims e quan son madurs son grogs, e a la rrail rredona en ssemblant de ceba marina...»). Para más información, cf. Grab-Kempf 2004: 298.

${ }^{31}$ Cf. por ejemplo la traducción al francés de los Comentarios de Mattioli (Mattiole, 1680: 242): «Ce que les Grecs appellent Dragontion, m'a été montré en trois especes...», traducción del latín (Matt. 1598: 446): «Id autem quod Graeci Dracontion vocant, triplici effigie mihi demonstratum est».

32 «Axarope para fazer saljr las ujruelas toma de la leche çinco onças lentigjs mude dramas vij dragontia iiij dramas. cuezelo todo en tres libras de agua fasta que torne a la terçia parte».

${ }_{33}$ «E tome çumo de puerro \& beuanlo con leche de muger \& destielle en el oydo o tome çumo de dragontia (\&) destillelo por el oydo tibio».

${ }^{34}$ Este vocablo también presenta testimonios en la versión catalana del Diccionario latino-hispano de Nebrija (Cfr. Soberanas, 1977: 160), al igual que en castellano (Nebrija 1545: f. 15r): «Aros, siue Arrhon, siue aron .u. el jarrillo yerua o draguntia»; además, este término es relativamente frecuente en latín.

${ }_{35}$ «Iten simjente de la draguntia beujda aguza la vista \& tira la manzilla rricardus». El texto latino del Thesaurus Pauperum que traduce, aunque con incorrecciones, es el siguiente (Da Rocha 1974: 117): «Item semen dragontee portatum uisum sanat. Kyrannus». Este misma receta aparece traducida en $S D M$ (f. 6r) con el nombre de «dragantea», en la versión castellana del Tesoro de los pobres (Hispano 1540: f. 6r), usando el término «tragontia» y en la versión aljamiada de este texto, con el nombre trigontina (Hespano 1999: 25).

${ }_{36}$ «Et la draguntia es buena paral que trae culuebra en el vientre dada a beuer sana».
} 
No he localizado testimonios en castellano de este término en relación con la botánica y que parece traducir el strignus editado por Kroemer (con la variante stringnus), definido como 'solatrum' en su glosario (1920: 81). El étimo más cercano es el estrin$g o^{37}$, que solo se recoge en Alonso y Ruyzes de Fontecha (1999) ${ }^{38}$ relacionado con dos plantas, el cacabum y el manicon: 'yerua que causa locura'. Parece tratarse, pues, de una variedad de las solanáceas, que también recibía las denominaciones de solatrum y strychnos (Daunay, Laterrot y Janick 2008: 24-25), términos ambos que confirman la definición de Kroemer. Se da la circunstancia de que un poco más adelante en este mismo capítulo figura la «uva bolpicia» (vid. infra), probable vulgarización del término uva vulpina que Leonhart Fuchs incluye bajo la denominación genérica De Strycno (1542: 691). Como se distinguían tradicionalmente cuatro especies de solatrum o solanum (denominados por Fuchs como hortense, halicácabo, somnífero y furioso), es difícil discernir si el estrinco se utiliza en la traducción castellana como denominación de una de las especies, como término genérico o como simple sinónimo de «uva bolpicia».

\section{GARNIGAL}

De las calientes en el terçero grado [...] De los maderos: genciana, polipodio, turbit, casia linea, azaro, garnigal, pimienta luenga, costo, çinamomi (83ra).

El garnigal aparece documentado en diversos lugares, en particular en los glosarios editados por Américo Castro, considerando el término un error de edición: «La transcripción de P. Meyer contiene numerosas erratas: galanga: garnigal por galanga ${ }^{39}$ : garingal» (1991: xii). No obstante, existen testimonios de este término en otros textos en francés ${ }^{40}$. En castellano figura en El cartulario del infante Luis de Navarra (Leroy 1981: 28), aunque es probable que se trate de un préstamo del francés: «1 libra de garnigal $=10$ sueldos». De ser así, habría que considerarlo un galicismo en el Libro de los grados. Tampoco se debe descartar una posible deturpación textual, como indica Castro para las transcripciones de Meyer, si bien ya parece menos probable que se hubiese producido la misma deturpación en dos textos distintos escritos en lenguas diferentes para referirse a la misma planta.

\footnotetext{
${ }^{37}$ Más cercano al latín, se documenta strigno (DETEMA).

${ }^{38}$ Apud RAE, Corpus diacrónico del español. http://www.rae.es (noviembre de 2012): «Cacabum, vna planta venenosa, estringo. [...] Manicon, vna especie de estringo, yerua que causa locura. Manicum, especie del solano, yerua».

${ }^{39}$ «Galanga, "planta exótica de raíz medicinal', del b. lat. galanga y este del ár. halánğ íd. $1 .{ }^{a}$ doc.: garengal, Alex.» (DCEH).

${ }^{40}$ Es el caso del Livre des simples medecines (Opsomer 1984: pp. 49, 164-165), identificado como el Kaempferia galanga. En Ordonnances des Roys de France (1721: 424) se lee: «Item. Cubebes, [...] fleur de canelle, garnigal, citonal, gales, gome...». Este término convive con garingal en Marguerite de Flandre. En la parte consagrada a las «especias» bajo el epígrafe Fruiterie de (Canat de Chizy 1859: 121) se recoge: «On y voit aussi la noix muguète, le macis, garnigal, tournesol, etc.; gironne, poivre long, poudre d'alixandre, graine de paradis, etc.». Sin embargo, en la indicación de gastos del 28 de diciembre (Canat de Chizy 1859: 253), se utiliza garingal: «Demi lt. de garingal, la lt. lx s., val. xxx s. iiij lt. de poudre d'Alixandre, la lt. xxiiij s., val. iiij 1. xvi d.».
} 


\section{MUZA}

De las que çierran [...] Asi son estas: pan de ceuada, puches, grano quebrantado, simul, datiles, niesperas, sizamo, avellotas, grosura de çieruo, e muza, que es arrope e vinagre compuesto (86rb).

Kroemer (1920: 78) indica en su glosario que el simple musa pertenece a la familia de las Musáceas, es decir, a alguna de las variedades del plátano. No obstante, los textos castellanos que presentan este término se inclinan más bien por un significado dentro del campo semántico de 'acerbo, agridulce', como también lo define el DETEMA ${ }^{41}$. El propio texto del Libro de los grados parece añadir una nueva acepción al introducir la siguiente glosa semántica: «que es arrope e vinagre compuesto». De esta manera, la muza podría interpretarse como un jarabe agridulce a base de fruta, lo que introduciría un compuesto en una lista de simples. Sin embargo, este término, junto con mus [t]a que figura en el capítulo de los simples húmedos en primer grado, parece hacer referencia a musa, unánime en el texto latino y que parece más acorde con un tratado que, fiel a su título, solo menciona medicamentos simples ${ }^{42}$. Por lo tanto, parece prudente considerar que la glosa semántica parte de un error de interpretación de este término por parte de algún copista y aceptar, a falta de nuevos elementos de análisis, la definición propuesta por Kroemer.

\section{OLIO UYLO POLEO}

De las atratiuas [...] petroleon, sulfur, armoniaco, †deçebrium $\dagger$, olio uylo, aristologia, raiz de caña, diptamo, tapsia, çiclamen, estiercol quemado, † $†$ seperillas $\dagger$ (85ra).

De las que çierran. Son aquellas que han (s)ter[re]stedat e son viscosas mayor mente aquellas que engendran vmores gruesos e espesos e viscosos. (86rb) Las melezinas que alinpian desoluiendo, asi como façe el calamento entrar molificando, asi como façe la casia fistola e maluas e con su terrestida[d] [o] [c]on viscosidad (86va).

La presencia de olio en este pasaje podría provenir de una deturpación de lolio ${ }^{43}$ para traducir el término latino lolium en un pasaje con numerosas divergencias con res-

${ }^{41}$ Carrera de la Red recoge diversos pasajes en los que se menciona el sustantivo Musa para definirlo como «una especie de fruto suave que se cria donde se crian las cañas del azucar» (Ruyzes). Asimismo, menciona a Font Quer (2002: 948), quien lo identifica con el plátano (Musa sapientum L.), al igual que Kroemer. Sin embargo, más adelante el Dictionarium medicum define el adjetivo Muzum como agro dulce, coincidiendo con el DETEMA. Cf. Nebrija (2001: 101 y 306).

${ }^{42}$ Este mismo argumento parece dificultar asimismo la posibilidad de que se trate del Musa trociscus o antídoto Musa, tradicionalmente usado para diversas afecciones (varían según la obra en que se cita) y compuesto por un mínimo de seis simples (cfr. Sabbah 1984: 109-123). Pablo de Egina hace referencia también a Antonius Musa en la obra de Galeno y alude a una cataplasma para el edema, compuesta de elaterio, estafisagria e hisopo (Adams 1844: 573). Más información sobre Antonius Musa en Michler (1993: 757-785).

${ }^{43}$ «Joyo, cizaña» DETEMA. Aunque este diccionario sólo cita un pasaje de CHS, el término lolio es utilizado por Andrés Laguna en varias ocasiones en su traducción de Dioscórides e incluso corrige las versiones latinas en el capítulo del moly (Diosc. III, 50) en el que traduce «harina de lolio» donde en latín dice «irino vnguento» (Matt., III, 47) o «trito cum yrino» en la versión longobarda (Stadler 1899: 399), que remite al lirio (Iris germanica L.). Para ello, Laguna retoma la argumentación de Mattioli en su commentario, quien afirma que el texto griego estaría deturpado y que tendría que decir «farina aerina, (hoc est lolii)» en concor- 
pecto al texto latino. En el mismo sentido, el uyló y su variante oyllo son los vocablos catalanes medievales utilizados con más frecuencia para traducir también el lolium ${ }^{44}$, lo cual podría establecer una nueva relación del texto castellano con la lengua catalana, que se sumaría a la ya citada del término argela. Tales vocablos, junto con el hipotético galicismo garnigal, podrían confirmar cuando menos una difusión textual inicial por el noreste de la Península Ibérica.

Una deturpación semejante a la de olio parece detectarse en el término poleo en Las meleçinas congelatiuas:

Las meleçinas congelatiuas $\cdot[\ldots]$ poleo, texa quemada, serpentaria, cas[c]a de hueuo, fieltro quemado (87rb).

Aunque es posible que la familia de la difusión textual de la que surgió la traducción romance presentase un hipotético pulegium, en la edición de Kroemer figura también el término lolium, que no encuentra equivalente castellano. Por último, el capítulo de las «calientes en el terçero grado» recoge igualmente lilio donde en latín figura lolio de forma unánime, lo cual indica que este simple latino parecía presentar algún tipo de problema en la transmisión al romance y dar lugar a frecuentes deturpaciones.

\section{TERRESTIDAD / TERRESTEDAT}

De las que çierran. Son aquellas que han (s)ter[re]stedat e son viscosas mayor mente aquellas que engendran vmores gruesos e espesos e viscosos. (86rb) Las melezinas que alinpian desoluiendo, asi como façe el calamento entrar molificando, asi como façe la casia fistola e maluas e con su terrestida[d][o] [c] on viscosidad (86va).

El CORDE $E^{45}$ recoge los términos terrestidat, terresteidad y terrestidad, definido este último por el DETEMA como «cualidad térrea». La presencia de terrestidad en tres ocasiones en $G O R^{46}$ y una en Alfonso de la Torre $(1991,220)^{47}$ parece avalar el término editado en el f. 86va y que aparece deturpado en el manuscrito. Por su parte, terrestidat figura en un único testimonio, en Gomez de Zamora (1995: f. 167v) ${ }^{48}$, al igual que terresteidad, que solo está presente en Diego de Santiago (1999: f. 82r) ${ }^{49}$. El

\footnotetext{
dancia con Galeno, quien describe el «Myle» siguiendo también al anazarbeo (Kühn, XII, 80): «26. De myle, mola [...] Nam cum farina lolii apposita, apertam vulvam sanare scribit Dioscorides». De hecho, la traducción moderna de Dioscórides al castellano indica «para el drenaje de la matriz, tras rallarla y aplicarla en supositorio vaginal con harina de cizaña» (López y Cortés: 2006: 239), sin más indicaciones al respecto.

${ }^{44}$ El más llamativo es la traducción catalana del capítulo LXIV «De Lolium» del Macer Floridus (Choulant: 1832: 112). La edición de Faraudo de Saint-Germain (1955-1956: 37) comienza: «De uyló. - Uyló és erba qi neix entre les messes».

${ }^{45}$ RAE: Banco de datos (CORDE) [en línea]. Corpus diacrónico del español. http://www.rae.es [consultado en noviembre de 2012].

${ }_{46}$ «(f. 15v) E sy fueren de melanconia: tornan a vna escuridad \& terrestidad [...] (25v) \& esto es porque puja mas enla melanconial la sequedad \& terrestidad [...] (148v) E sy la color negra que es por quemamiento torna a clareza: \& si es por melanconia torna a terrestidad [...] pero el venino poniendo lo en agua va se al fondon por su terrestidad \& globosidad \& mayor quemamiento \& ençenizamiento».

47 «E sy fuere la terrestidad ynmunda, fazer se ha dende plomo e antymonio».

48 «Aqueso es uerdat quel tal del caso del pecado ligera mente le leuantan e(n)l qual enla terrestidat suya piensa \& que por consideraçion \& omjldat la gracia terrenal... »

${ }^{49}$ (f. 82r) «se consuma la umidad al fuego o al sol, y quedará la sal limpia de la terresteidad».
} 
vocablo terrestridad, mucho más común, figura ya en el Diccionario de Autoridades y luego en los diccionarios de la $R A E$ hasta la edición de 1822 y en otros repertorios hasta $1869^{50}$. Por el contrario, no he localizado testimonios de terrestedad, terrestredad o terresteda $t^{51}$, por lo que la edición de este último vocablo constituye una mera hipótesis de trabajo, con base en la interpretación del manuscrito, en los testimonios citados y en la fuente latina, que utiliza los términos terrestria ${ }^{52}$ y terestritate ${ }^{53}$.

\section{UVA BOLPICIA}

De las secas en el segundo grado. [...] estrinco, catapucia, ciperis, mestranto, sisilion, uva bolpicia, aneldo, puerros, coriandro (84ra).

En un capítulo particularmente complejo del Libro de los grados, no existe equivalente en el texto latino para la expresión «uva bolpicia», por lo que podría tratarse de un desplazamiento textual, un añadido posterior, un sinónimo de estrinco (vid. supra) o una simple deturpación en una familia concreta de la fuente latina o de la difusión en romance. Aunque en el manuscrito se lee claramente «vna bonjcia», es verosímil que este simple provenga de alguna de las variedades conocidas como «uva vulpina» $\mathrm{u}$ otras denominaciones equivalentes, razón por la cual la edición adopta de forma hipotética bolpicia ${ }^{54}$.

El Proyecto Anthos atribuye denominaciones cercanas a esta a dos plantas de su corpus. En primer lugar, el Sedum album L., que recibe el nombre popular de uvas de raposo en gallego y portugués, aunque no en castellano (Colmeiro II: 459; Font Quer 2002: 297). Font Quer considera que esta especie podría corresponder con el Sedum minus de Dioscórides, que Laguna (Diosc. IV, 92) denomina «otra especie de Siempre viua» y la define diciendo que es seca en segundo grado, al igual que la «uva bolpicia» objeto de análisis. También se utiliza en castellano el denominativo uva de raposa y uva de zorra para la Paris quadrifolia L. (Colmeiro V: 94; Font Quer 2002: 905 y ss.). Además, Andrés Laguna indica en su comentario al dorycnio (Diosc. VI, 6) que los árabes denominaban uva de raposo al solatro furioso, planta venenosa cuyo jugo se utilizaba para emponzoñar las lanzas y que podría hacer referencia a la belladona. No obstante, no se encuentra ninguna referencia al zorro en los nombres vernáculos de esta planta en Colmeiro (IV: 150) ni en Font Quer (2002: 563) ni en el Proyecto Anthos, aunque sí la denominación solano furioso. Las únicas indicaciones que Laguna aporta

\footnotetext{
${ }^{50}$ Para datos más concretos a este respecto, cfr. NTLLE.

${ }^{51}$ El DIEC2 define terrestridat: 'condició de terrestre, de terrenal'; el DCVB presenta, además la variante «terrestreitat», que considera incorrecta.

${ }_{52}^{5}$ La tesis de De la Rosa Cubo presenta un estudio y edición de la obra Summa Medicinae atribuida a Arnau de Vilanova, si bien la propia editora afirma en varias ocasiones que tal atribución es falsa (2000: 38,70 y ss.). Ciertos pasajes de esta obra presentan textos cercanos al lib. de simpl. med. virt.. Concretamente, en el capítulo opilativa, que presenta párrafos paralelos al De opilativis editado por Kroemer, el Pseudo-Arnau utiliza el término terrestreitatem donde el lib. de simpl. med. virt. usa «terrestria», sin variantes en las versiones colacionadas por Kroemer.

${ }^{53}$ La versión que Kroemer denomina Ysaac presenta la variante terestreitate.

${ }^{54}$ A pesar de que no he localizado este vocablo ni el latino vulpitia, la edición respeta el manuscrito en la parte final de la palabra, cuyas últimas tres letras son idénticas al cercano catapucia.
} 
para su identificación son que esta planta es diversa del dorycnio y que «es una frigidisima especie de yerua Mora». Ahondando en esta denominación, la hierba mora se utiliza para designar cuatro plantas en castellano, entre las que se encuentran el Solanum nigrum, especie citada por Daunay, Laterrot y Janick (2008: 24), a la que atribuyen, entre otras, la denominación de Uva vulpis. Por su parte, el Liber aggregatus (1525, ff. 156v) describe el «solatro» («De solatro .i. vua vulpina id est solatrum») entre las plantas frías y secas en segundo grado ${ }^{55}$. Esta circunstancia, unida a la descripción del Sedum minus de Laguna, indica que el espectro de plantas que aquí se estudian poseía un temperamento semejante en la tradición médica y podría ser indicio de que la presencia de la uva bolpicia en el Libro de los grados, más que una deturpación textual, debería considerarse un añadido posterior y consciente de algún copista latino o castellano que poseía un sólido conocimiento de fitoterapia.

\section{REFERENCIAS BIBLIOGRÁFICAS}

ADAMS, Francis, ed. (1844): The seven books of Paulus Aegineta: Translated from the Greek, I-3, Londres, The Sydenham Society.

Alcover, Antoni $\mathrm{M}^{\mathrm{a}}$ y Francesc de B. Moll (1993): Diccionari Català-Valencià-Balear, Palma de Mallorca, Moll. (DCVB)

ALEMANY y Bolufer, José (1917): Diccionario de la Lengua Española, Barcelona, Ramón Sopena.

AMIGÓ ANGLÈs, Ramón (1999): Introducció a la recerca en toponímia i antroponímia, Barcelona, L'Abadia de Montserrat.

ANDRÉ, Jacques (1967): Les noms d'oiseaux en latin, Paris, Librairie C. Klincksieck.

AYDILlO SAN MARTíN, Julián (2006): Pueblos y apellidos de España: diccionario etimológico, Alicante, Editorial Club Universitario.

Beaujouan, Guy (1972): «Manuscrits médicaux du Moyen âge conservés en Espagne», Mélanges de la Casa de Velázquez, 8 [reimpr. en París, IDEM, Science médievale d'Espagne et d'alentour, Variorum, Aldershot, 1992].

BENSOUSSAN, Maurice (2002): Les particules alimentaires: naissance de la gastronomie au XVIe siècle de François Ier à la colonisation de l'Amérique du nord, Paris, Masonneuve y Larose.

BRU DE RAMÓN, Juan Bautista (1784): Colección de láminas que representan los animales y monstruos del Real Gabinete de Historia Natural de Madrid, Madrid, Imprenta de Andrés de Sotos.

CANAT DE ChIZY, Marcel (1859): «Marguerite de Flandre, duchesse de Bourgogne. Sa vie intime et l'état de sa maison (Extrait des escroes de la dépense de son hôtel)», Mémoires de l'Académie Impériale des Sciences, Arts et Belles-Lettres de Dijon., segunda serie, t. VII, Dijon, Imprimerie Rabutot.

${ }^{55}$ El Liber de gradibus solo hace referencia al strigno, con el mismo temperamento (Israelí 1515: f. 82r). Por su parte, Serapión, que distingue tres especies de solatrum, no menciona el estrigno (1525, ff. 115r). 
CARBÓN, Damián (1997): Libro del arte de las comadres o madrinas y del regimiento de

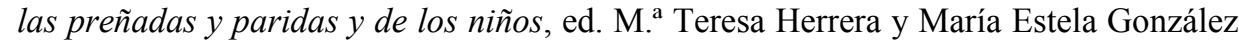
de Fauve, Madison, Hispanic Seminary of Medieval Studies. (CBN)

CAStro, Américo (1991): Glosarios latino-españoles de la Edad Media, Madrid, CSIC.

Choulant, Ludwig (ed.) (1832): Macer Floridus, De Viribus Herbarum, Leipzig, Leopold Voss.

COLMEIRO PENIDO, Miguel (1885-1889): Enumeración y revisión de las plantas de la Península Hispano-Lusitana é islas Baleares, vols. I-V, Madrid, Imprenta de la viuda é hija de Fuentenebro.

Constantino el Africano (1536): Liber de gradibus, Basilea, Henricus Petrus.

Coromines, Joan y J. A. PASCUAL (1980-1991): Diccionario crítico etimológico castellano e hispánico, Madrid, Gredos (DCEH).

Corpus diacrónico del español. http://www.rae.es (consultado en mayo de 2012).

CORTÉS, Jerónimo (1672): Libro y tratado de los animales terrestres y volátiles, con la historia y propriedades dellos, Valencia, Juan Chrysósotomo Gárriz [1. a ed. de 1613].

DAUNAY, Marie-Christine, Henri LATERROT y Jules JANICK (2008): «Iconography and History of Solanaceae: Antiquitiy to the $17^{\text {th }}$ Century», Horticultural Reviews, 34.

DH. REAL ACADEMIA ESPAÑOLA (1960-1996): Diccionario histórico de la lengua española, apud Nuevo diccionario histórico de la lengua española, recurso on-line. URL: http://web.frl.es/dh.html.

DíAZ GONZÁlEZ, Olga Josefina (1986): El habla de Candamo: aspectos morfosintácticos, Oviedo, Univ. de Oviedo.

DiosCóRIDES LATINO, ed. lib. I por K. Hofmann-T.M. Auracher, «Der Longobardische Dioskorides des Marcellus Virgilius», Romanische Forschungen, 1, 1883, pp. 49-105; lib. II-V por $\mathrm{H}$.

FARAUdo DE SAINT GERMAIN, Lluís (1955-1956): «Una versió catalana del libre de les herbes de Macer», Estudis Romanics, 5, pp. 1-54.

FonT Quer, Pío $\left(2002^{4}\right)$ : Plantas medicinales. El Dioscórides renovado, Barcelona, ed. Península.

FradeJas RuedA, José Manuel (2002): La caza de la Edad Media, Valladolid, Universidad de Valladolid, Instituto de Estudios de Iberoamérica y Portugal.

FuCHS, Leonhart (1542): De historia stirpium comentarii insignes, Basilea, Oficinia Isingriniana.

GARCÍA ARIAS, Xosé Lluis (2007): Propuestes etimolóxiques, vol. 2, col. Llibrería llingüística, vol. 18, Uvieu, Academia Llingua Asturiana.

GARCíA DE DIEGO, Vicente $\left(1978^{3}\right)$ : Dialectología española, Madrid, Ediciones Cultura Hispánica del Centro Iberoamericano de Cooperación.

García GonZÁleZ, Alejandro (2012): «El Breviarium de Iohannes de Sancto Paulo y la materia médica en el manuscrito E.IV.22 de la Real Biblioteca de El Escorial», en José Martínez Gázquez, Oscar de la Cruz Palma, Cándida Ferrero Hernández, eds., Estudios de Latín Medieval Hispánico: Actas del V Congreso Hispánico de Latín Medieval, Barcelona, 7-10 de septiembre de 2009, Florencia, Sismel, pp. 533-556.

GESNER, Konrad (1555): Historiae animalium liber III, Tiguri apud Christ. Froschover.

GilberTo (2002): El libro de recetas, Madrid, Real Biblioteca 3063, ed. lsabel Zurrón, Madison, Hispanic Seminary of Medieval Studies $(G I L)$.

GLICK T. F., Livesey S. y F. WALLIS (2005): Medieval science, technology, and medicine : an encyclopedia, New York, Routledge. 
GomeZ De Zamora, Alfonso (1992): Morales de Ovidio. BNM ms. 10144, ed. Derek C. Carr, Madison, Hispanic Seminary of Medieval Studies.

Gordonio, Bernardo de (1989): Lilio de medicina, ed. John Cull y Cynthia Wasick, Madison, Hispanic Seminary of Medieval Studies. (GOR)

GRAB-KEMPF Elke (2004): «Etymologische Notizen $\mathrm{zu}$ einem altkatalanischen Übersetzungstext: Abenhuefidi (Ibn Wāfid), Libre de les Medicines Particulars», Zeitschrift für romanische Philologie, 120, 2, pp. 282-311.

GuTIÉRREZ RodiLla, Bertha M. (2010): «Sobre lexicografía médica del renacimiento castellano: los vocabularios de Andrés Laguna y Bartolomé Hidalgo de Agüero», Revista de Lexicografia, 16, pp. 59-74.

HEIDER, Gustav (1851): «Physiologus, nach einer Handschrift des XI. Jahrhunderts», Archiv für österreichische Geschichte, 5, pp. 541-582.

Herrera, M ${ }^{\mathrm{a}}$ Teresa (dir.) (1996): Diccionario español de textos médicos antiguos, Madrid, Arco/Libros. (DETEMA).

— ed. (1987): Tratado médico (Tratado de la patología general), Madison, Hispanic Seminary of Medieval Studies. (TRM)

—

- y M. ${ }^{\text {a }}$ Estela GONZÁLEZ DE FAUVE, ed. (1997): Traducción del Tratado de cirugía de Guido de Cauliaco, Madison, Hispanic Seminary of Medieval Studies. (CAU)

Hespano, Pedro (1999): Tesoro de los proves: versão em judeu-castelhano aljamiado (séc. $X V)$, ed. Maria Adélia Soares de Carvalho Mendes, Porto, Fundação Eng. António de Almeida.

HISPÀ, Pere (1892): Tresor de pobres compilat per Pere Hispano (Papa Joan XXI), versió catalana del segle XIV, ed. Josep Balari, Barcelona, Impr. Fidel Giró.

HisPano, Pedro (1540): Libro de medicina llamado Tesoro de los pobres, con un regimiento de sanidad, Sevilla, Juan Cromberger.

IBN WAFID (1943): El 'Libre de les medicines particulars' versión catalana trescentista del texto árabe del tratado de los medicamentos simples de Ibn Wáfid, autor médico toledano del siglo XI, ed. Lluis Faraudo de Saint-Germain, Barcelona, Real Academia de Buenas Letras.

InSTITUT D'EstUdis CATALANS, Diccionari de la llengua catalana, segona edició. URL: http://dlc.iec.cat/ (DIEC2)

IsIDORO DE SEVILla (1994): Etimologías I-II (edición bilingüe), ed. José Oroz Reta y Manuel A. Marcos Casquero, Madrid, BAE ( $2^{\mathrm{a}}$ edición).

ISAAC ISRAELI, Omnia opera Ysaac, Lyon, Bartholomeus Trott, 1515. (1984): Tratado de las fiebres, ed. Ruth M. Richards, Madison, Hispanic Seminary of Medieval Studies. (FIE)

JuAna Aranzana, Eduardo de y Juan M. VAREla (2000): Guía de las aves de España: Península, Baleares y Canarias, Barcelona, Lynx edicions.

Ketham, Johannes de (1990): Compendio de la humana salud, ed. María Teresa Herrera, Madrid, Arco/Libros (CHS).

KROEMER, Georg Heinrich (ed.): Johanns von Sancto Paulo, "Liber de simplicium medicinarum virtutibus» und ein anderer Salernitaner Traktat, "Quae medicinae pro quibus morbis donandae sunt» nach dem Breslauer Codex herausgegeben, Borna-Leipzig, Druck von Robert Noske, 1920.

KÜHN, C. G., ed. (1821-1833): Claudii Galeni, Opera Omnia (I-XX), Leipzig, Car. Cnoblochii. 
LEROY, Béatrice, ed. (1981): El Cartulario del infante Luis de Navarra del año 1361, Pamplona, Institución Príncipe de Viana, Diputación Floral de Navarra.

LÓPEZ DE AyAla, Pero (1986): Libro de la caça de las aves (ms 16.392, British Library, Londres), ed. John G. Cummins, Londres, Tamesis.

LÓPEZ EIRE, Antonio, Francisco CORTÉS GABAUdAN y Bertha GuTIÉRREZ RodiLla (2006): Dioscórides, sobre los remedios medicinales, manuscrito de Salamanca, estudios y traducción, Salamanca, Universidad de Salamanca.

MALlENT, Joseph (1788): Encyclopedia metódica. Historia natural de las aves, tomo I, Madrid, Antonio Sancha.

MARTín FERreira A. I. y A. GARCÍA GONZÁlez (2010): «La tradición manuscrita del Brevarium de Johannes de Sancto Paulo», Exemplaria Classica, Journal of Classical Philology, 14, pp. 227-248.

Matthiole, Pierre A. (1680): Les Commentaires de C.P. André Matthiole,... sur les six livres de la matière médicinale de Pedacius Dioscoride,... traduits de latin en françois par C. Antoine Du Pinet, Lyon, J. B. de Ville.

Mattioli, Petri A. (1598): Opera quae extant omnia, hoc est Commentarii in VI libros Pedacii Dioscoridis Anazarbei de medica materia, adjectis in margine variis graeci textus lectionibus, Francofurti, ex officina typographica N. Bassaei.

Michler, M. (1993): «Principis medicus: Antonius Musa», Aufstieg und Niedergang der Römischen Welt, Berlin, Walter de Gruyter, pp. 757-785.

Montero Cartelle, Enrique (1983): Constantini Liber de coitu, Santiago de Compostela, Universidad de Santiago.

NeBriJA, Antonio de (1536): Dictionarium Ael. Antonii Nebrissensis, Granada.

- (2001): Dictionarium Medicum, ed. Avelina Carrera de la Red, Salamanca, Univ. de Salamanca.

Ordonnances des roys de France de la troisième race (1729), volume II, París, Imprimerie Royale.

PENSADO TOMÉ, José Luis (1974): Opúsculos lingüísticos gallegos del siglo XVIII, Vigo, Galaxia.

Plinio (1938-1962): Natural History, 10 vols., Londres / Cambridge, Massachusetts, (reimpr. 1967-1989).

Real ACADEmia Española (2001): Diccionario de la lengua española, 22a edición, Madrid, Espasa Calpe (DRAE).

- (2001): Nuevo tesoro lexicográfico de la lengua española, Espasa Calpe, Madrid (edición en CD-ROM y en línea: < http://buscon.rae.es/ntlle/SrvltGUILoginNtlle >) (NTLLE).

ReAl JARDín BotÁnico y CSIC, Proyecto Anthos, http://www.anthos.es (consultado en noviembre de 2012).

Rocha Pereira, Ma Helena da (1972): Obras médicas de Pedro Hispano, Coimbra, Acta Universitatis Conimbrigensis.

Rosa CubO, C. de la (2000): Summa medicinae - Mad. Esc. M. II. 17. Estudio y edición crítica, Tesis Doctoral, Valladolid, Universidad de Valladolid.

RuYZES DE FONTECHA, Juan, (1999): Diez privilegios para mujeres preñadas, ed. M. ${ }^{\text {a }}$ Purificación Zabía Lasala, Madrid, Arco/Libros.

SABBAH, Guy (1984): «Une composition médicale d'Antonius Musa dans les textes MÉDICAUX latins tardifs», Mémoires V, Textes Médicaux Latins Antiques, Saint-Étienne, Centre Jean Palerne, Publications de l'Univ. de Saint-Etienne, pp. 109-124. 
SANTAMARÍA HernándeZ, M. ${ }^{a}$ Teresa (2009): «La tradición de la toxicología grecolatina en el Herbario de Ps. Apuleyo: el capítulo sobre la hierba basilisca», en Arsenio Ferraces Rodríguez, ed., Fito-zooterapia antigua y altomedieval: textos y doctrinas, A Coruña, Universidade da Coruña, pp. 97-119.

SANTIAGO, Diego de (1999): Arte separatoria y modo de apartar todos los licores..., ed. de Alegría Alonso González, Salamanca, CILUS, 1999 (apud CORDE, consultado en noviembre de 2012).

SARTON G. y E. GILPATRICK (1922): «Twelfth Critical Bibliography of the History and Philosophy of Science and of the History of Civilization (to March 1922)», Isis, 4, 3, pp. 572-647.

SERAPIÓN, J, (1525): «Liber Serapionis aggregatus in medicinis simplicibus», en Practica Jo. Serapiones, Lyon, per Jacobum Myt, ff.113-201v.

SoberanAS, Amadeu-J. (1977): «Les edicions catalanes del Diccionari de Nebrija», en Germà Colon, ed., Actes del quart col-loqui internacional de llengua i literatura, Barcelona, Publicacions de l'Abadia de Montserrat, pp. 141-204.

STADLER H. (1897): «Dioscorides Longobardus (Cod. Lat. Monacensis 337)», Romanische Forschungen, 10, pp. 181-247 y 369-446.

SteEle, Robert (ed.) (1920): Opera hactenus inedita Rogeri Baconi Fasc. V, Secretum Secretorum..., Londres, Oxford University Press.

TORRE, Alfonso de la (1991): Visión deleytable, ed. Jorge García López, Universidad de Salamanca (apud CORDE, consultado en noviembre de 2012).

VermeIlle, Alexandre (2006): Physiologus. De l'Orient à l'Occident. Un patchwork au service de l'Écriture, texto online. URL: http: <www.summagallicana.it> (consultado en noviembre de 2012). 